\title{
Systematic Review \\ Effect of Coffee Consumption on Non-Alcoholic Fatty Liver Disease Incidence, Prevalence and Risk of Significant Liver Fibrosis: Systematic Review with Meta-Analysis of Observational Studies
}

\author{
Maryam Ebadi *, Stephen Ip, Rahima A. Bhanji (D) and Aldo J. Montano-Loza * \\ Division of Gastroenterology \& Liver Unit, University of Alberta Hospital, Edmonton, AB T6G 2X8, Canada; \\ sip@ualberta.ca (S.I.); rbhanji@ualberta.ca (R.A.B.) \\ * Correspondence: ebadi@ualberta.ca (M.E.); montanol@ualberta.ca (A.J.M.-L.); \\ Tel.: +780-248-1892 (M.E. \& A.J.M.-L.); Fax: +780-248-1895 (M.E. \& A.J.M.-L.)
}

Citation: Ebadi, M.; Ip, S.; Bhanji, R.A.; Montano-Loza, A.J. Effect of Coffee Consumption on

Non-Alcoholic Fatty Liver Disease Incidence, Prevalence and Risk of Significant Liver Fibrosis: Systematic Review with Meta-Analysis of Observational Studies. Nutrients 2021, 13, 3042. https://doi.org/10.3390/ nu13093042

Academic Editors: Naoki Tanaka and Antoni Sureda

Received: 6 July 2021

Accepted: 27 August 2021

Published: 30 August 2021

Publisher's Note: MDPI stays neutral with regard to jurisdictional claims in published maps and institutional affiliations.

Copyright: (c) 2021 by the authors. Licensee MDPI, Basel, Switzerland. This article is an open access article distributed under the terms and conditions of the Creative Commons Attribution (CC BY) license (https:// creativecommons.org/licenses/by/ $4.0 /)$.

\begin{abstract}
Background and aim: Non-alcoholic fatty liver disease (NAFLD) is the most common cause of chronic liver disease worldwide. Given the anti-fibrotic and antioxidant properties of coffee, this systematic review and meta-analysis aims to provide updated results on the impact of coffee consumption on NAFLD incidence, prevalence, and risk of significant liver fibrosis. Methods: We conducted a comprehensive search in MEDLINE (OvidSP) and Scopus from January 2010 through January 2021. Relative risks for the highest versus the lowest level of coffee consumption were pooled using random-effects models. Heterogeneity and publication bias were evaluated using the Higgins' $I^{2}$ statistic and Egger's regression test, respectively. Results: Eleven articles consisting of two case-control studies, eight cross-sectional studies, and one prospective cohort study were included in the meta-analysis. Of those, three studies with 92,075 subjects were included in the analysis for NAFLD incidence, eight studies with 9558 subjects for NAFLD prevalence, and five with 4303 subjects were used for the analysis of liver fibrosis. There was no association between coffee consumption and NAFLD incidence (RR $0.88,95 \%$ CI $0.63-1.25, p=0.48$ ) or NAFLD prevalence (RR $0.88,95 \%$ CI $0.76-1.02, p=0.09$ ). The meta-analysis showed coffee consumption to be significantly associated with a $35 \%$ decreased odds of significant liver fibrosis (RR 0.65, 95\% CI 0.54-0.78, $p<0.00001)$. There was no heterogeneity $\left(I^{2}=11 \%, p=0.34\right)$ and no evidence of publication bias $(p=0.134)$. Conclusion: This meta-analysis supports the protective role of coffee consumption on significant liver fibrosis in patients with NAFLD. However, the threshold of coffee consumption to achieve hepatoprotective effects needs to be established in prospective trials.
\end{abstract}

Keywords: coffee; caffeine; NAFLD; liver fibrosis

\section{Introduction}

Non-alcoholic fatty liver disease (NAFLD) is the most common cause of chronic liver disease in North America and is one of the main health complications seen around the world. Recently, a change in its nomenclature to Metabolic-Associated Fatty Liver Disease (MAFLD) [1,2] has been proposed by a group of experts. This acronym seems to better capture pathogenesis but has not been widely accepted. In this review, we will continue to use NAFLD.

The estimated prevalence of NAFLD is $25-30 \%$ in the adult population [3] and it is a leading indication for liver transplantation [4]. NAFLD constitutes a histological spectrum starting with lipid accumulation in hepatocytes (simple steatosis) without significant inflammation or liver fibrosis, with the potential to progress to non-alcoholic steatohepatitis (NASH) (varying stages of fibrosis) and subsequently to cirrhosis development with an increased risk of decompensation and hepatocellular carcinoma (HCC) [5]. Worsening fibrosis is the main factor associated with liver-related morbidity and mortality in patients 
with NAFLD [6]. NAFLD is frequently diagnosed as an incidental finding based on abnormal serum liver function tests and "bright liver" on imaging or hepatomegaly $[7,8]$ in subjects without significant alcohol consumption and with no other cause of chronic liver disease.

Genetic and lifestyle factors play an important role in the pathogenesis of NAFLD. Thus, current management focuses mainly on lifestyle adaptations to reduce steatosis and risk of fibrosis development. Numerous studies have shown beneficial effects of coffee consumption in various diseases including diabetes mellitus type 2, cardiovascular disease, HCC, and NAFLD [9-11]. The anti-fibrotic and cytoprotective antioxidant properties of coffee may inhibit onset of hepatic steatosis or progression to steatohepatitis with fibrosis $[12,13]$. Natural polyphenols, present in coffee, have been shown to protect the liver from fibrosis, which is a major surrogate for negative clinical outcomes in NASH [14]. In experimental studies, decaffeinated coffee attenuated liver steatosis, oxidative stress, and inflammation in rats on a high-fat diet [15]. However, coffee consumption has no impact on the serum levels of an inflammatory mediator, leptin, in patients with mild to severe hepatic steatosis [16].

Nevertheless, the effects of coffee consumption on NAFLD risk have been conflicting. Therefore, this systematic review and meta-analysis aims to provide updated results on the impact of coffee consumption on NAFLD incidence, prevalence, and liver fibrosis risk.

\section{Material \& Methods}

\subsection{Study Search and Selection}

We conducted a comprehensive search for titles, abstracts, and keywords in MEDLINE (OvidSP) and Scopus using the search term: (coffee OR caffeine) combined with (NASH OR NAFL* OR "nonalcoholic fatty" OR "nonalcoholic steatohepatitis" OR "liver steatosis" OR "hepatic steatosis" OR "steatohep" OR "hepatic fibrosis" OR "liver fibrosis" OR "hepatosteatosis") from January 2010 through January 2021. Reference lists of included manuscripts and published meta-analyses were also checked manually. The search was limited to human studies written in English.

The inclusion criteria were as follows: (a) case-control, cross-sectional, or cohort studies; (b) published as original studies to evaluate the impact of coffee consumption on NAFLD incidence, prevalence, and risk of significant liver fibrosis; (c) including relative risks (RRs), odds ratios (ORs), or hazard ratios (HRs) with 95\% confidence intervals ( $95 \%$ CIs) or adequate data to calculate them; (d) NAFLD diagnosis by serum biomarkers of liver injury or imaging studies (i.e., ultrasound, computed tomography, etc.), elastography, or liver histological evaluation.

We excluded studies without sufficient statistics or adequate data for risk estimates, lack of biochemical tests and imaging to evaluate liver injury, no description of coffee intake measurement (e.g., questionnaire, dietary recall, food record), use of coffee derivatives, no discrimination between coffee or caffeine as the main exposure, or if NAFLD incidence, prevalence, or significant liver fibrosis was not the main outcome of interest. Studies evaluating the association between coffee consumption and NAFLD in the general population or in patients with NAFLD are included. We consider significant liver fibrosis as a histological score greater than or equal to F2 according METAVIR or elastography $>8 \mathrm{kPa}$. All these studies assessed liver fibrosis in a single time-point and therefore, we present the results as liver fibrosis associated with NAFLD but not the fibrosis progression in NAFLD, as assessing progression requires more than one-time measurements.

\subsection{Data Extraction}

For each study, the author names and year of publication, data on study design, population, NAFLD diagnosis, coffee consumption assessment, steatosis and fibrosis severity classification, RRs/HRs/ORs estimates for extreme categories of coffee intake and corresponding 95\% CIs, the adjustments for confounding variables, and main findings were extracted. We did not communicate with the study authors regarding missing information. 
The methodological quality of each article was assessed using the Newcastle-Ottawa Scale (NOS) [17], which assesses each study in three areas including the selection of participants and measurement of exposure, the comparability of study groups, and evaluation of outcome. The adapted Newcastle-Ottawa scale [18], giving a maximum of five stars for selection, two stars for comparability, and three stars for outcome, was applied for crosssectional studies. Assessment of study eligibility and quality was conducted independently by ME and AML, and any discrepancies regarding the inclusion/exclusion of studies or data extraction were determined by a consensus of the reviewers.

\subsection{Statistical Analysis}

In this meta-analysis, ORs of the case-control and the cross-sectional studies as well as HRs of the cohort study were used as an estimate of RRs. RRs for the highest versus the lowest level of coffee consumption were pooled using random-effects models. If more than one RR estimate was reported, the one most comprehensively adjusted for potential confounding factors was used. For studies reporting only the number of cases and controls for each category of coffee consumption, crude RRs and the corresponding 95\% CIs were computed from these frequencies. Studies with risk estimates of two categories (e.g., coffee drinkers versus non-drinkers) were also integrated.

Heterogeneity was evaluated using the Higgins' $I^{2}$ statistic with values $\leq 25 \%, \leq 50 \%$, $\leq 75 \%$, and $>75 \%$ representing no, small, moderate, and significant heterogeneity, respectively [19]. We also examined the stability of the results by performing a sensitivity analysis, in which we calculated pooled RRs and $95 \%$ CIs after excluding one study at a time from the analysis. Potential publication bias was assessed using Egger's regression test [20] with the evidence of small-study effects on $\mathrm{P}_{\text {Egger }}<0.1$. All analyses were performed using Stata 16.0 (StataCorp, https: / /www.stata.com) and Review Manager (Rev-Man) version 5.4 with $p$-values $<0.05$ considered as statistically significant.

\section{Results}

\subsection{Publication Selection and Quality Assessment}

The initial search strategy yielded 303 potentially relevant articles after the exclusion of duplicates. Two hundred and sixty-seven articles were excluded after screening titles and abstracts, leaving the remaining 36 articles for a full-text article review. According to inclusion and exclusion criteria, 11 articles fulfilled the eligibility criteria and, therefore, were included in the data analysis [11,16,21-29]. The detailed flow chart of the selection of eligible literature included in the meta-analysis is presented in Figure 1. Included studies are presented in Table 1. These 11 articles consist of two case-control studies, eight crosssectional studies, and one prospective cohort study. From these studies, three studies with 92,075 subjects [21-23] were included in the analysis for NAFLD incidence, eight studies with 9558 subjects for NAFLD prevalence [11,16,23-28], and five studies with 4303 subjects were used for the analysis of significant liver fibrosis risk [11,23,28,29].

Data on coffee consumption was collected by interviewer-administrated questionnaire in three studies $[23,25,29]$. The severity of hepatic steatosis was identified using liver ultrasonography in six studies [16,23-27] while elastography [28] and biopsy [11] were performed in two studies. Fibrosis severity was determined based on liver histology in two studies [11,29]. Four articles had a score of seven stars [16,22,27,29], six with eight stars [11,21,23,24,26,28], and only one article was nine stars [25] by NOS. Reporting items for systematic reviews and meta-analysis are included as Supplementary Materials (Supplementary Table S1). 


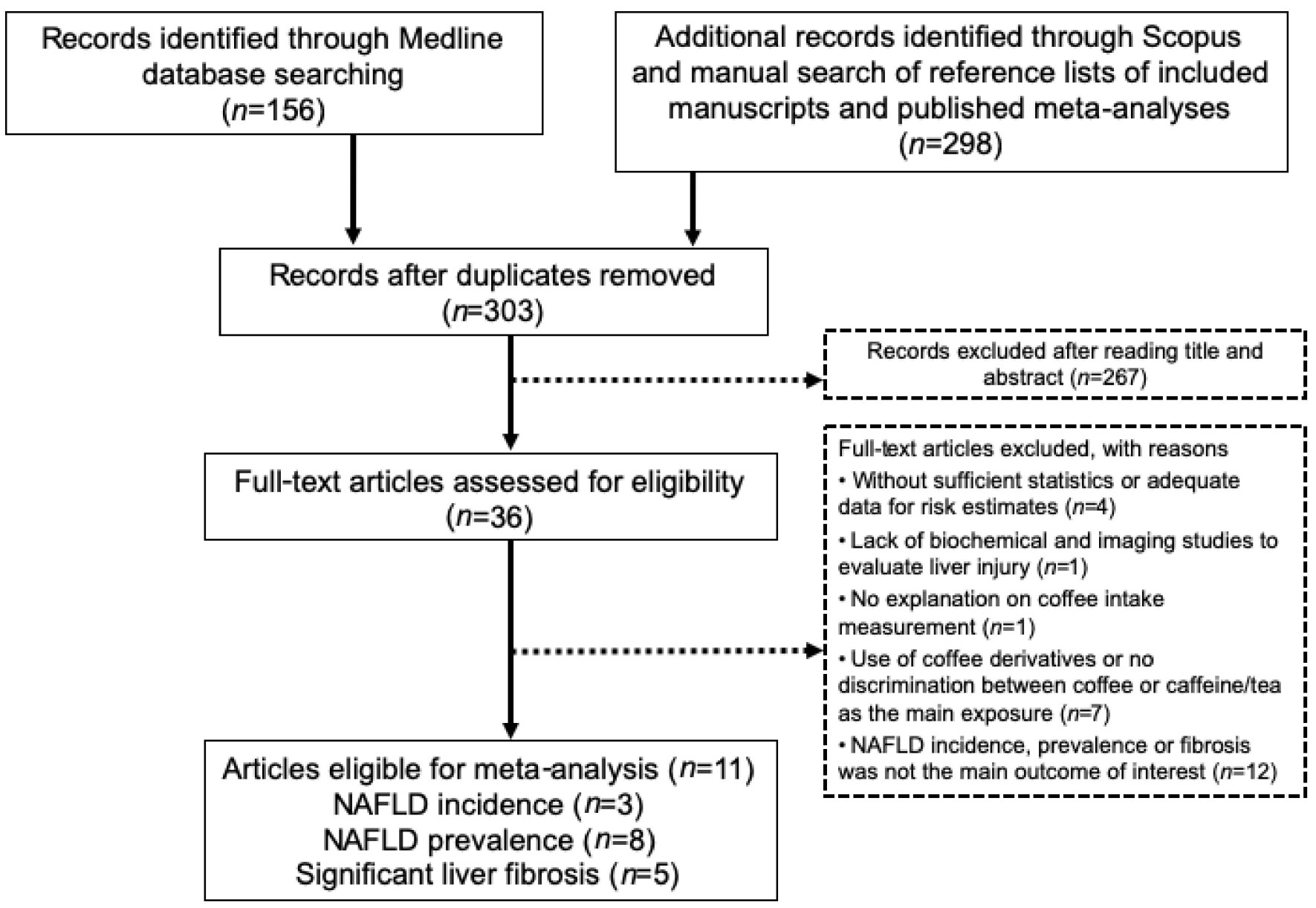

Figure 1. Flow chart of literature search and inclusion of studies in the meta-analysis. Abbreviation: NAFLD, Non-alcoholic fatty liver disease. 
Table 1. Summary of studies investigating the impact of coffee consumption on NAFLD incidence, prevalence, and liver fibrosis.

\begin{tabular}{|c|c|c|c|c|c|c|c|c|c|c|}
\hline Study & Study Design & Population & $\begin{array}{l}\text { NAFLD } \\
\text { Diagnosis }\end{array}$ & $\begin{array}{l}\text { Steatosis } \\
\text { Severity }\end{array}$ & $\begin{array}{l}\text { Fibrosis } \\
\text { Severity }\end{array}$ & $\begin{array}{l}\text { Coffee Intake } \\
\text { Measurement }\end{array}$ & Main Finding & $\begin{array}{l}\mathrm{RR} / \mathrm{HR} / \mathrm{OR} \\
\text { Estimates for } \\
\text { Extreme } \\
\text { Categories of } \\
\text { Coffee Intake } \\
(95 \% \mathrm{CI})\end{array}$ & $\begin{array}{l}\text { Adjustments for } \\
\text { Confounding } \\
\text { Variables }\end{array}$ & NOS \\
\hline $\begin{array}{l}\text { Chung et al., } \\
2020 \text { [21] }\end{array}$ & $\begin{array}{l}\text { Retrospective } \\
\text { longitudinal } \\
\text { cohort }\end{array}$ & $\begin{array}{c}91,436 \text { subjects participating in a } \\
\text { comprehensive health-screening } \\
\text { program were followed for a mean of } \\
2.8 \text { years, } 13,362 \text { (15\% developed } \\
\text { fatty liver) }\end{array}$ & Ultrasound & $\begin{array}{c}\text { Steatosis } \\
\text { incidence: } \\
\text { increase in liver } \\
\text { echogenicity } \\
\text { compared with } \\
\text { the renal cortex } \\
\text { echogenicity }\end{array}$ & & $\begin{array}{l}\text { Self-administered } \\
\text { food frequency } \\
\text { questionnaire }\end{array}$ & $\begin{array}{l}\text { No association } \\
\text { between fatty liver } \\
\text { incidence and the } \\
\text { amount of coffee } \\
\text { consumption at } \\
\text { baseline }\end{array}$ & $\begin{array}{l}\text { HR } 1.09 \\
(0.97-1.22)\end{array}$ & $\begin{array}{l}\text { Age, sex, education, } \\
\text { exercise, smoking, } \\
\text { alcohol intake, centre } \\
\text { and year, BMI, total } \\
\text { energy intake, } \\
\text { triglyceride, LDL-C, } \\
\text { HDL-C, glucose, } \\
\text { alanine } \\
\text { aminotransferase, } \\
\text { aspartate } \\
\text { aminotransferase, } \\
\text { change of alcohol, } \\
\text { change of BMI, and } \\
\text { change of exercise }\end{array}$ & 8 \\
\hline $\begin{array}{l}\text { Zhang et al., } \\
2020 \text { [28] }\end{array}$ & Cross-sectional & $\begin{array}{l}555 \text { NAFLD patients from } \\
\text { multi-centre hepatology clinics }\end{array}$ & $\begin{array}{l}\text { Radiological } \\
\text { features, liver } \\
\text { histology, or } \\
\text { elevated alanine } \\
\text { aminotrans- } \\
\text { ferase or } \\
\text { aspartate amino- } \\
\text { transferase } \\
\text { levels with a } \\
\text { CAP } \\
\text { value } \geq 248 \mathrm{~dB} / \mathrm{m}\end{array}$ & $\begin{array}{l}\text { Elastography: } \\
\text { severe hepatic } \\
\text { steatosis: CAP } \\
\text { threshold of } \\
280 \mathrm{~dB} / \mathrm{m}\end{array}$ & $\begin{array}{l}\text { Elastography: } \\
\text { advanced } \\
\text { fibrosis: LSM } \\
\text { threshold of } \\
10 \mathrm{kPa}\end{array}$ & $\begin{array}{l}\text { Standardized, } \\
\text { self-administered } \\
\text { questionnaires }\end{array}$ & $\begin{array}{c}\text { Inverse } \\
\text { association } \\
\text { between coffee } \\
\text { consumption and } \\
\text { advanced fibrosis } \\
\text { No association } \\
\text { between coffee } \\
\text { consumption and } \\
\text { severe hepatic } \\
\text { steatosis }\end{array}$ & $\begin{array}{c}\text { OR } 0.49 \\
(0.27-0.88) \\
p=0.02 \\
\text { OR } 0.92 \\
(0.59-1.44) \\
p=0.71\end{array}$ & $\begin{array}{l}\text { Age, sex, smoking and } \\
\text { alcohol status, coffee, } \\
\text { tea, and soft drinks } \\
\text { drinker, time spent on } \\
\text { different physical } \\
\text { activities, energy } \\
\text { expenditure, physical } \\
\text { activity level and } \\
\text { meeting physical } \\
\text { activity guidelines (\%), } \\
\text { obesity and type } 2 \\
\text { diabetes, and } \\
\text { participating centre }\end{array}$ & 8 \\
\hline
\end{tabular}


Table 1. Cont

\begin{tabular}{|c|c|c|c|c|c|c|c|c|c|c|}
\hline Study & Study Design & Population & $\begin{array}{l}\text { NAFLD } \\
\text { Diagnosis }\end{array}$ & $\begin{array}{l}\text { Steatosis } \\
\text { Severity }\end{array}$ & $\begin{array}{l}\text { Fibrosis } \\
\text { Severity }\end{array}$ & $\begin{array}{l}\text { Coffee Intake } \\
\text { Measurement }\end{array}$ & Main Finding & $\begin{array}{l}\mathrm{RR} / \mathrm{HR} / \mathrm{OR} \\
\text { Estimates for } \\
\text { Extreme } \\
\text { Categories of } \\
\text { Coffee Intake } \\
(95 \% \mathrm{CI}) \\
\end{array}$ & $\begin{array}{l}\text { Adjustments for } \\
\text { Confounding } \\
\text { Variables }\end{array}$ & NOS \\
\hline $\begin{array}{l}\text { Veronese et al., } \\
\quad 2018[24]\end{array}$ & Cross-sectional & $\begin{array}{l}2819 \text { randomly sampled participants } \\
\text { from electoral rolls } \\
\text { Absence of fatty liver in } 1627 \text { subjects } \\
\text { and } 916 \text { with NAFLD (134 patients } \\
\text { with Liver Steatosis Score of 6) }\end{array}$ & Ultrasound & $\begin{array}{l}\text { Fatty liver score } \\
\text { ranged from } 0 \text { to } 6 \text {, } \\
\text { with higher values } \\
\text { indicating a } \\
\text { greater severity }\end{array}$ & & $\begin{array}{l}\text { Self-reported } \\
\text { validated } \\
\text { semi-quantitative } \\
\text { food frequency } \\
\text { questionnaire }\end{array}$ & $\begin{array}{l}\text { No association } \\
\text { between coffee } \\
\text { consumption and } \\
\text { lower odds of } \\
\text { liver steatosis }\end{array}$ & $\begin{array}{l}\text { OR } 0.97 \\
(0.71-1.32) \\
p=0.84\end{array}$ & $\begin{array}{c}\text { Age, sex, smoking } \\
\text { status, presence of } \\
\text { diabetes, gastric ulcer, } \\
\text { cancer, acute } \\
\text { myocardial infarction, } \\
\text { waist circumference, } \\
\text { systolic and diastolic } \\
\text { blood pressure, daily } \\
\text { energy, and alcohol } \\
\text { intake }\end{array}$ & 8 \\
\hline $\begin{array}{l}\text { Alferink et al., } \\
2017 \text { [25] }\end{array}$ & Cross-sectional & $\begin{array}{l}\text { Population-based cohort of } 2424 \\
\text { participants ( } 35 \% \text { steatosis) }\end{array}$ & Ultrasound & $\begin{array}{c}\text { Presence or } \\
\text { absence of a } \\
\text { hyper-echogenic } \\
\text { liver parenchyma }\end{array}$ & $\begin{array}{l}\text { Transient } \\
\text { elastography: } \\
\text { liver stiffness } \\
\text { measurements } \\
(\mathrm{LSM}) \geq 8.0 \mathrm{kPa}\end{array}$ & $\begin{array}{l}\text { Validated 389-item } \\
\text { food frequency } \\
\text { questionnaire }\end{array}$ & $\begin{array}{c}\text { Independent } \\
\text { association } \\
\text { between frequent } \\
\text { coffee } \\
\text { consumption and } \\
\text { lower probability } \\
\text { of significant liver } \\
\text { fibrosis } \\
\text { No significant } \\
\text { association } \\
\text { between coffee } \\
\text { intake and } \\
\text { steatosis }\end{array}$ & $\begin{array}{c}\text { OR } 0.39 \\
(0.18-0.86) \\
p=0.005 \\
\text { OR } 1.15 \\
(0.75-1.77) \\
p=0.192\end{array}$ & $\begin{array}{l}\text { Tea, energy intake, } \\
\text { BMI, gender, age, } \\
\text { steatosis, alanine } \\
\text { aminotransferase, } \\
\text { excessive alcohol } \\
\text { intake, current or } \\
\text { former smoking and } \\
\text { HOMA-IR, soda } \\
\text { consumption, cream } \\
\text { and sugar use, dietary } \\
\text { quality, and physical } \\
\text { activity } \\
\text { Tea, energy intake, } \\
\text { BMI, gender, age, } \\
\text { HOMA-IR, excessive } \\
\text { alcohol intake, current } \\
\text { or former smoking }\end{array}$ & 9 \\
\hline
\end{tabular}


Table 1. Cont

\begin{tabular}{|c|c|c|c|c|c|c|c|c|c|c|}
\hline Study & Study Design & Population & $\begin{array}{c}\text { NAFLD } \\
\text { Diagnosis }\end{array}$ & $\begin{array}{l}\text { Steatosis } \\
\text { Severity }\end{array}$ & $\begin{array}{l}\text { Fibrosis } \\
\text { Severity }\end{array}$ & $\begin{array}{l}\text { Coffee Intake } \\
\text { Measurement }\end{array}$ & Main Finding & $\begin{array}{l}\mathrm{RR} / \mathrm{HR} / \mathrm{OR} \\
\text { Estimates for } \\
\text { Extreme } \\
\text { Categories of } \\
\text { Coffee Intake } \\
\text { (95\% CI) }\end{array}$ & $\begin{array}{l}\text { Adjustments for } \\
\text { Confounding } \\
\text { Variables }\end{array}$ & NOS \\
\hline $\begin{array}{l}\text { Katsagoni et al., } \\
\quad 2017 \text { [27] }\end{array}$ & Case-control & $\begin{array}{l}100 \text { newly ultrasound-proven } \\
\text { NAFLD patients ( } 21 \text { with NASH), } 55 \\
\text { healthy controls matched for age, sex, } \\
\text { and BMI }\end{array}$ & $\begin{array}{c}\text { Elevated } \\
\text { alanine amino- } \\
\text { transferase } \\
\text { and/or gamma- } \\
\text { glutamyl } \\
\text { transpeptidase } \\
\text { levels, } \\
\text { elastography, } \\
\text { and evidence of } \\
\text { hepatic steatosis } \\
\text { on ultrasound } \\
\text { (available for 85) } \\
\text { and/or } \\
\text { compatible liver } \\
\text { histology }(n= \\
\text { 32) }\end{array}$ & $\begin{array}{l}\text { Evidence of } \\
\text { hepatic steatosis at } \\
\text { ultrasonography } \\
\text { Biopsy: NASH } \\
\text { Diagnosis: } \\
\text { NAFLD Activity } \\
\text { score (NAS) } \geq 5\end{array}$ & & $\begin{array}{l}\text { Semi-quantitative } \\
\text { validated food } \\
\text { frequency } \\
\text { questionnaire }\end{array}$ & $\begin{array}{c}\text { Inverse } \\
\text { association } \\
\text { between coffee } \\
\text { intake and } \\
\text { NAFLD presence } \\
\text { (OR 0.68, 95\% CI } \\
0.49-0.94, p=0.02) \\
\text { Lost its } \\
\text { significance once } \\
\text { adjusted for } \\
\text { adiponectin and } \\
\text { TNF-a }\end{array}$ & $\begin{array}{c}\text { OR } 0.72 \\
(0.49-1.04) \\
p=0.07\end{array}$ & $\begin{array}{c}\text { Age, sex, waist } \\
\text { circumference, } \\
\text { HOMA-IR, } \\
\text { adiponectin, and } \\
\text { TNF-a }\end{array}$ & 7 \\
\hline $\begin{array}{l}\text { Zelber-Sagi } \\
\text { et al., 2015 [23] }\end{array}$ & $\begin{array}{l}\text { Cross-sectional } \\
\text { and prospective } \\
\text { cohort }\end{array}$ & $\begin{array}{c}\text { Cross-sectional cohort } \\
\text { 347 general population, 31\% } \\
\text { diagnosed with NAFLD } \\
\text { Prospective cohort } \\
\text { A subgroup of patients without fatty } \\
\text { liver at baseline who were followed } \\
\text { up for 7 years } \\
(n=147)\end{array}$ & $\begin{array}{l}\text { Ultrasound } \\
\text { and SteatoTest }\end{array}$ & $\begin{array}{l}\text { Hepatorenal index } \\
\text { (HRI) on US, } \\
\text { NashTest } \\
\text { (borderline NASH } \\
\text { or definite NASH) } \\
\text { SteatoTest }(\geq 5 \% \text {, } \\
\quad \geq \text { S1-S2) }\end{array}$ & $\begin{array}{c}\text { FibroTest } \\
\text { (significant } \\
\text { fibrosis }(\geq \mathrm{F} 2) \text { ) }\end{array}$ & $\begin{array}{c}\text { Interviewer- } \\
\text { administrated } \\
\text { questionnaire and } \\
\text { detailed } \\
\text { semiquantitative } \\
\text { food-frequency } \\
\text { questionnaire }\end{array}$ & $\begin{array}{c}\text { Cross-sectional } \\
\text { cohort: } \\
\text { Inverse } \\
\text { association } \\
\text { between coffee } \\
\text { consumption and } \\
\text { significant liver } \\
\text { fibrosis and no } \\
\text { association with } \\
\text { the } \\
\text { development of } \\
\text { steatosis } \\
\text { Prospective } \\
\text { cohort: } \\
\text { No association } \\
\text { between coffee } \\
\text { consumption and } \\
\text { NAFLD incidence }\end{array}$ & $\begin{array}{c}\text { NAFLD } \\
\text { prevalence } \\
0.92(0.57-1.50), \\
p=0.75 \\
\text { Hepatic } \\
\text { Fibrosis:0.49 } \\
(0.25-0.97), \\
p=0.04 \\
\text { NAFLD } \\
\text { Incidence: } \\
0.72(0.28-1.85), \\
p=0.501\end{array}$ & $\begin{array}{c}\text { Current smoking, } \\
\text { sugar intake, physical } \\
\text { activity (minutes per } \\
\text { week), serum } \\
\text { cholesterol levels, and } \\
\text { dietary fat and calories } \\
\text { intake } \\
\text { Current smoking, } \\
\text { sugar intake, physical } \\
\text { activity }\end{array}$ & 8 \\
\hline
\end{tabular}


Table 1. Cont.

\begin{tabular}{|c|c|c|c|c|c|c|c|c|c|c|}
\hline Study & Study Design & Population & $\begin{array}{c}\text { NAFLD } \\
\text { Diagnosis }\end{array}$ & $\begin{array}{l}\text { Steatosis } \\
\text { Severity }\end{array}$ & $\begin{array}{l}\text { Fibrosis } \\
\text { Severity }\end{array}$ & $\begin{array}{l}\text { Coffee Intake } \\
\text { Measurement }\end{array}$ & Main Finding & $\begin{array}{c}\mathrm{RR} / \mathrm{HR} / \mathrm{OR} \\
\text { Estimates for } \\
\text { Extreme } \\
\text { Categories of } \\
\text { Coffee Intake } \\
(95 \% \mathrm{CI})\end{array}$ & $\begin{array}{l}\text { Adjustments for } \\
\text { Confounding } \\
\text { Variables }\end{array}$ & NOS \\
\hline $\begin{array}{l}\text { Imatoh et al., } \\
2015 \text { [16] }\end{array}$ & Cross-sectional & $\begin{array}{l}1024 \text { Japanese male workers } \\
\text { receiving annual health checkups } \\
\text { Non-steatosis }(n=270) \text { Steatosis } \\
\qquad(n=754)\end{array}$ & Ultrasound & $\begin{array}{l}\text { No, mild, or } \\
\text { moderate-to- } \\
\text { severe hepatic } \\
\text { steatosis }\end{array}$ & & $\begin{array}{l}\text { Self-reported } \\
\text { questionnaire }\end{array}$ & $\begin{array}{l}\text { Dose-dependent } \\
\text { protective effect of } \\
\text { coffee on the } \\
\text { prevalence of } \\
\text { hepatic steatosis }\end{array}$ & $\begin{array}{c}\text { OR } 0.59 \\
(0.38-0.90) \\
p=0.03\end{array}$ & $\begin{array}{l}\text { BMI, age, smoking } \\
\text { status, alcohol } \\
\text { drinking, and green tea } \\
\text { consumption }\end{array}$ & 7 \\
\hline $\begin{array}{l}\text { Graeter et al., } \\
2015 \text { [26] }\end{array}$ & Cross-sectional & $\begin{array}{l}\text { Random population-based sample } \\
\text { with } 1452 \text { subjects ( } 381 \text { diagnosed } \\
\text { with hepatic steatosis) }\end{array}$ & Ultrasound & $\begin{array}{l}\text { No steatosis and } \\
\text { steatosis grade I, II } \\
\text { and III }\end{array}$ & & $\begin{array}{l}\text { Standardized } \\
\text { questionnaire }\end{array}$ & $\begin{array}{l}\text { No association } \\
\text { between hepatic } \\
\text { steatosis and } \\
\text { coffee } \\
\text { consumption }\end{array}$ & $\begin{array}{l}\text { OR } 0.77 \\
(0.44-1.34) \\
p=0.81\end{array}$ & $\begin{array}{l}\text { Age, BMI, gender, } \\
\text { metabolic syndrome, } \\
\text { and physical activity }\end{array}$ & 8 \\
\hline $\begin{array}{l}\text { Bambha et al., } \\
2014 \text { [11] }\end{array}$ & Cross-sectional & $\begin{array}{c}782 \text { biopsy-proven NAFLD patients; } \\
\text { Advanced fibrosis (>stage 2) in } 25 \% \\
(n=199) \\
\text { NASH (definite or probable): in } 79 \% \\
(n=616)\end{array}$ & Biopsy & $\begin{array}{l}\text { Presence versus } \\
\text { absence of NASH } \\
\text { histology ((1) } \\
\text { definite } \\
\text { steatohepatitis; (2) } \\
\text { definitely not } \\
\text { steatohepatitis; } \\
\text { and (3) borderline } \\
\text { steatohepatitis) }\end{array}$ & $\begin{array}{l}\text { None to } \\
\text { moderate } \\
(\leq \text { Stage } 2) \text { or } \\
\text { advanced } \\
(>\text { Stage } 2)\end{array}$ & $\begin{array}{c}\text { Self-reported } \\
\text { validated dietary } \\
\text { questionnaire }\end{array}$ & $\begin{array}{c}\text { Significant } \\
\text { association } \\
\text { between coffee } \\
\text { intake and } \\
\text { decreased odds of } \\
\text { advanced fibrosis } \\
\text { in patients with } \\
\text { lower HOMA-IR }\end{array}$ & $\begin{array}{l}\text { OR } 0.68 \\
(0.52-0.89) \\
p=0.005\end{array}$ & $\begin{array}{c}\text { Age, sex, race, waist } \\
\text { circumference, } \\
\text { aspartate transaminase, } \\
\text { gamma-glutamyl } \\
\text { transferase diabetes, } \\
\text { smoking, alcohol, } \\
\text { biopsy length, } \\
\text { HOMA-IR, and } \\
\text { interaction between } \\
\text { coffee and HOMA-IR }\end{array}$ & 8 \\
\hline
\end{tabular}


Table 1. Cont.

\begin{tabular}{|c|c|c|c|c|c|c|c|c|c|c|}
\hline Study & Study Design & Population & $\begin{array}{c}\text { NAFLD } \\
\text { Diagnosis }\end{array}$ & $\begin{array}{l}\text { Steatosis } \\
\text { Severity }\end{array}$ & $\begin{array}{l}\text { Fibrosis } \\
\text { Severity }\end{array}$ & $\begin{array}{l}\text { Coffee Intake } \\
\text { Measurement }\end{array}$ & Main Finding & $\begin{array}{c}\mathrm{RR} / \mathrm{HR} / \mathrm{OR} \\
\text { Estimates for } \\
\text { Extreme } \\
\text { Categories of } \\
\text { Coffee Intake } \\
(95 \% \mathrm{CI})\end{array}$ & $\begin{array}{l}\text { Adjustments for } \\
\text { Confounding } \\
\text { Variables }\end{array}$ & NOS \\
\hline $\begin{array}{c}\text { Anty et al., } 2012 \\
\text { [29] }\end{array}$ & Cross-sectional & $\begin{array}{l}195 \text { severely and morbidly obese } \\
\text { patients, referred for bariatric surgery } \\
\text { of which NASH was present in 19.5\% }\end{array}$ & Biopsy & $\begin{array}{l}\text { NAFLD activity } \\
\text { score (NAS) } \\
\text { simple steatosis } \\
\text { (NAS } \leq 2 \text { ), } \\
\text { borderline } \\
\text { (3 } \leq \text { NAS } \leq 4) \text {, or } \\
\text { definitive NASH } \\
\text { (NAS } \geq 5 \text { ) }\end{array}$ & $\begin{array}{c}\text { Significant } \\
\text { fibrosis }(F \geq 2)\end{array}$ & $\begin{array}{l}\text { Interviewer- } \\
\text { administered } \\
\text { questionnaire }\end{array}$ & $\begin{array}{l}\text { Regular coffee } \\
\text { consumption was } \\
\text { an independent } \\
\text { protective factor } \\
\text { for significant } \\
\text { fibrosis }\end{array}$ & $\begin{array}{c}\text { OR: } 0.752 \\
(0.578-0.980) \\
p=0.04\end{array}$ & $\begin{array}{c}\text { Aspartate } \\
\text { aminotransferase, } \\
\text { presence of NASH, } \\
\text { presence of the } \\
\text { metabolic syndrome, } \\
\text { and level of HOMA-IR }\end{array}$ & 7 \\
\hline $\begin{array}{l}\text { Funatsu et al., } \\
2011 \text { [22] }\end{array}$ & $\begin{array}{c}\text { Nested } \\
\text { case-control }\end{array}$ & $\begin{array}{l}1236 \text { subjects followed for } 5 \text { years; of } \\
\text { those, } 164 \text { males with fatty liver were } \\
\text { matched (age, BMI, and exercise } \\
\text { level) with } 328 \text { without fatty liver }\end{array}$ & Ultrasound & $\begin{array}{c}\text { Steatosis } \\
\text { incidence: } \\
\text { a bright liver, an } \\
\text { increase in the } \\
\text { liver-kidney } \\
\text { contrast, and/or a } \\
\text { decrease in liver } \\
\text { deep echo }\end{array}$ & & $\begin{array}{l}\text { Self-administered } \\
\text { questionnaire }\end{array}$ & $\begin{array}{l}\text { Daily coffee intake } \\
\text { was inversely } \\
\text { associated with } \\
\text { fatty liver } \\
\text { development }\end{array}$ & $\begin{array}{c}0.74(0.61-0.89) \\
p=0.001\end{array}$ & $\begin{array}{l}\text { Age, BMI, exercise, } \\
\text { daily alcohol intake, } \\
\text { and changes in BMI, } \\
\text { exercise level, and } \\
\text { daily alcohol intake } \\
\text { over time }\end{array}$ & 7 \\
\hline
\end{tabular}

Abbreviation: BMI, body mass index; HR, hazard ratio; NAFLD, non-alcoholic fatty liver disease; NASH, alcoholic steatohepatitis; NOS, the Newcastle-Ottawa Scale; OR, odds ratio; RR, relative risk. 


\subsection{Coffee Consumption and NAFLD}

Association between coffee consumption and NAFLD incidence, prevalence, and liver fibrosis risk was investigated in previous observational studies. In a cross-sectional study of 347 subjects, an inverse association was reported between coffee consumption ( 3 cups/day) and degree of liver fibrosis, but there was no association with NAFLD prevalence. Similarly, when a subgroup of subjects without fatty liver at baseline $(n=147)$ was prospectively followed for 7 years, no association was found between coffee intake and NAFLD onset assessed by liver ultrasound (OR 0.72, 95\% CI 0.28-1.85; $p=0.50$ ). Although poorer lifestyles were noticed in subjects with high coffee intake (three cups/day), controlling for lifestyle factors (smoking, diet, and physical activity) did not reveal any association with NAFLD onset [23]. No association was found between the amount of coffee consumption at baseline and NAFLD incidence in a cohort of 91,436 subjects who were followed for a mean of 2.8 years; however, an increase in the amount of coffee consumption, rather than the absolute amount, was associated with reduced NAFLD incidence (HR 0.94, 95\% CI 0.90-0.98) [21].

A recent cross-sectional study in 555 NAFLD patients found no association between coffee drinking and severe hepatic steatosis, defined as CAP $280 \mathrm{~dB} / \mathrm{m}$. Coffee consumption was associated with a lower risk of advanced fibrosis (defined as liver stiffness measurement (LSM) $\geq 10 \mathrm{kPa}$ ) after adjusting for confounders (OR $0.49,95 \%$ CI $0.27-0.88$; $p=0.02$ ) [28]. Similarly, an Italian study demonstrated that coffee drinking may not be protective against the severity of hepatic steatosis. In a cross-sectional study of middle-aged Italian participants, higher coffee intake was not associated with a lower risk of NAFLD, or severity of liver steatosis when adjusted for age, sex, smoking status, presence of diabetes, cancer, acute myocardial infarction, waist circumference, blood pressure, daily energy, and alcohol intake [24].

Increased intake of coffee, rather than total caffeine intake from other sources, was protective against the progression of fibrosis in patients with NASH. In a cross-sectional study of biopsy-proven NASH patients and ultrasound-negative controls, coffee intake was higher in controls and patients with NASH stage 0-1 compared to those with stage 2-4 fibrosis, suggesting the beneficial effects of coffee in preventing fibrosis development [9]. The metabolic background of patients might be an important determinant of coffee effect on liver fibrosis, as an inverse association between coffee intake and advanced fibrosis was only observed among patients with NAFLD and low insulin resistance (HOMA-IR <4.3), with no protective effect in patients with higher HOMA-IR [11].

A dose-dependent protective effect of coffee on the prevalence [16] and severity of hepatic steatosis [30] was reported in previous studies. More than three cups of coffee per day was associated with a lower risk of NAFLD when compared to intake of $<2$ cups per day [31].

\subsection{Meta-Analysis}

\subsubsection{Coffee Consumption and NAFLD Incidence}

During the three to seven years' follow up, no association was seen between coffee consumption and NAFLD incidence in subjects without NAFLD at baseline (RR 0.88, 95\% CI 0.63-1.25, $p=0.48$; Figure 2a). There was significant heterogeneity $\left(I^{2}=83 \%\right.$, $p=0.003)$ but no evidence of publication bias $(p=0.674)$. In a sensitivity analysis that excluded the study by Chung et al. [21], a reduction in heterogeneity $\left(I^{2}=0 \%, p=0.96\right)$ was associated with a significant reduction in the incidence of NAFLD (RR 0.74, 95\% CI 0.61, $0.89, p=0.002$ ). Due to the small number of studies, we were unable to conduct subgroup analyses to determine the sources of heterogeneity. However, NAFLD has a prolonged natural course, and variable follow-up times between studies ranging from 2.8 years in the study by Chung et al. [21] to 7 years in a study by Zelber-Sagi [23] may explain some of the heterogeneity. 


\section{a) NAFLD Incidence}

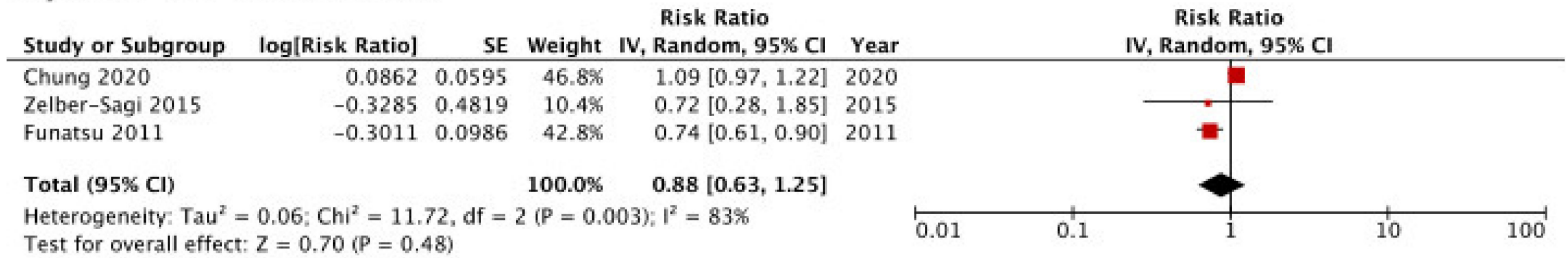

\section{b) NAFLD Prevalence}

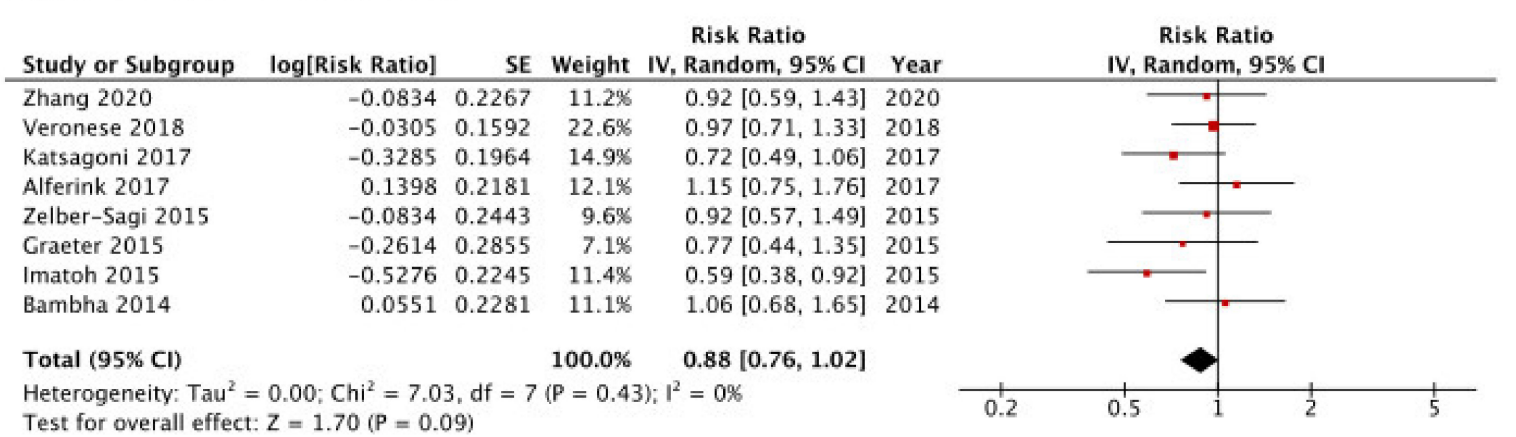

\section{c) Significant Liver Fibrosis}

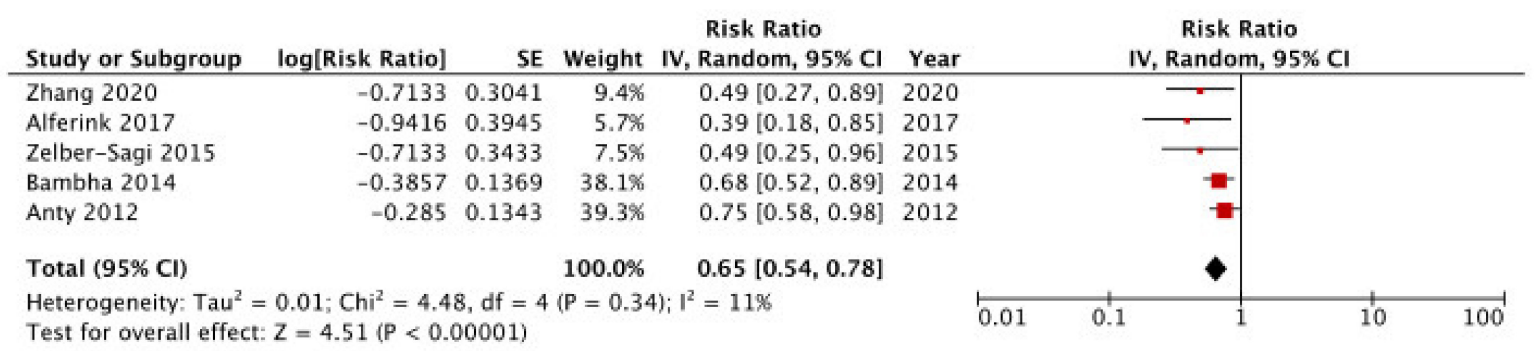

Figure 2. Forest plot for the association between coffee consumption and (a) NAFLD incidence, (b) NAFLD prevalence, and (c) significant liver fibrosis. Relative risk for the highest versus the lowest level of coffee consumption was pooled using random-effects models.

\subsubsection{Coffee Consumption and NAFLD Prevalence}

A total of one case-control [27] and seven cross-sectional [11,16,23-26,28] studies were pooled to evaluate the association between coffee consumption and NAFLD prevalence. The analysis revealed no significant association between coffee consumption and NAFLD prevalence (RR 0.88, 95\% CI 0.76-1.02, $p=0.09$; Figure 2b). There was no evidence of heterogeneity $\left(I^{2}=0 \%, p=0.43\right)$ or publication bias $(p=0.699)$. To check the effect of individual studies on the pooled RR, a sensitivity analysis was performed with exclusion of one trial [25] (Alferink et al. 2017), resulting in a significant reduction in the risk of NAFLD prevalence by coffee consumption (RR 0.85, 95\% CI 0.72-0.99, $p=0.04$ ). There was no significant change in RR or $I^{2}$ values with removal of other studies. Subgroup analyses according to the study design, questionnaire administration, study quality, and energy intake adjustment revealed that the association was significant in studies with lower quality, without adjustment for total energy intake, or when the self-reported questionnaire was applied (Table 2a). 
Table 2. Subgroup meta-analysis of coffee consumption and (a) NAFLD prevalence, (b) significant liver fibrosis stratified by study design, questionnaire administration, study quality, and energy intake adjustment.

\begin{tabular}{|c|c|c|c|}
\hline Subgroup & Number of Studies & Pooled RR & Heterogeneity \\
\hline \multicolumn{4}{|l|}{ NAFLD Prevalence } \\
\hline All studies & 8 & $0.88(0.76-1.02), p=0.09$ & $I^{2}=0 \%, p=0.43$ \\
\hline \multicolumn{4}{|l|}{ Study design } \\
\hline Case-control & 1 & $0.72(0.49-1.06), p=0.09$ & \\
\hline Cross-sectional & 7 & $0.91(0.77-1.07), p=0.25$ & $I^{2}=0 \%, p=0.44$ \\
\hline \multicolumn{4}{|l|}{ Questionnaire } \\
\hline Self-reported & 6 & $0.84(0.71-0.99), p=0.04$ & $I^{2}=3 \%, p=0.40$ \\
\hline Interviewed & 2 & $1.04(0.76-1.43), p=0.80$ & $I^{2}=0 \%, p=0.50$ \\
\hline \multicolumn{4}{|l|}{ NOS score } \\
\hline 7 & 2 & $0.66(0.49-0.88), p=0.005$ & $I^{2}=3 \%, p=0.50$ \\
\hline $8-9$ & 6 & $0.97(0.82-1.16), p=0.76$ & $I^{2}=0 \%, p=0.91$ \\
\hline \multicolumn{4}{|l|}{ Energy Intake Adjustment } \\
\hline Yes & 2 & $1.03(0.80-1.32), p=0.82$ & $I^{2}=0 \%, p=0.53$ \\
\hline No & 6 & $0.81(0.67-0.97), p=0.02$ & $I^{2}=0 \%, p=0.50$ \\
\hline \multicolumn{4}{|c|}{ Significant Liver Fibrosis } \\
\hline All studies & 5 & $0.65(0.54-0.78), p<0.00001$ & $I^{2}=11 \%, p=0.34$ \\
\hline \multicolumn{4}{|l|}{ Questionnaire } \\
\hline Self-reported & 2 & $0.64(0.50-0.82), p=0.0004$ & $I^{2}=0 \%, p=0.33$ \\
\hline Interviewed & 3 & $0.60(0.40-0.89), p=0.01$ & $I^{2}=42 \%, p=0.18$ \\
\hline \multicolumn{4}{|l|}{ NOS score } \\
\hline 7 & 1 & $0.75(0.58-0.98), p=0.03$ & \\
\hline $8-9$ & 4 & $0.60(0.48-0.75), p<0.00001$ & $I^{2}=0 \%, p=0.42$ \\
\hline \multicolumn{4}{|l|}{ Energy Intake Adjustment } \\
\hline Yes & 2 & $0.44(0.27-0.74), p=0.002$ & $I^{2}=0 \%, p=0.66$ \\
\hline No & 3 & $0.69(0.58-0.83), p<0.0001$ & $I^{2}=0 \%, p=0.43$ \\
\hline \multicolumn{4}{|l|}{ Population } \\
\hline NAFLD patients & 2 & $0.64(0.50-0.82), p=0.0004$ & $I^{2}=0 \%, p=0.33$ \\
\hline General population & 3 & $0.60(0.40-0.89), p=0.01$ & $I^{2}=42 \%, p=0.18$ \\
\hline
\end{tabular}

Abbreviation: NAFLD, non-alcoholic fatty liver disease; NOS, the Newcastle-Ottawa Scale; RR, relative risk.

\subsubsection{Coffee Consumption and Risk of Significant Liver Fibrosis}

Meta-analysis with a randomized-effect model including five studies showed that coffee consumption was significantly associated with $35 \%$ decreased odds of liver fibrosis (RR 0.65, 95\% CI 0.54-0.78, $p<0.00001$; Figure 2c) with no heterogeneity $\left(I^{2}=11 \%, p=0.34\right)$. Egger's linear regression test was performed and showed no publication bias $(p=0.134)$. Sensitivity analysis by excluding one study at a time revealed high stability of the results and no single influential study on the pooled RR, which varied from 0.59 (95\% CI 0.43-0.80) when the study from Bambha et al. [11] was excluded to 0.68 (95\% CI 0.57-0.80) when the study from Alferink et al. [25] was removed. Results did not change after performing a subgroup analysis based on study design, questionnaire administration, study quality, and energy intake adjustment (Table 2b). Results were also stable in both NAFLD patients (RR $0.64,95 \%$ CI $0.50-0.82, p=0.0004$ ) and the general population (RR 0.60, 95\% CI 0.40-0.89, $p=0.01)$.

\section{Discussion}

Association between coffee consumption and NAFLD incidence, prevalence, and significant liver fibrosis risk was quantitatively assessed in our meta-analysis. We found increased coffee consumption was significantly associated with a reduced risk of significant liver fibrosis. This association was robust and consistent between different subgroups 
and in our sensitivity analysis. However, no association was observed between coffee consumption and NAFLD incidence or prevalence.

In the majority of studies, liver steatosis and fibrosis were not quantified histologically and were diagnosed based on imaging studies. The sensitivity of ultrasound in revealing hepatic steatosis varies between 60 to $94 \%$ depending on the population of the interest [32], and ultrasonography can only detect steatosis with $>2.5-20 \%$ liver fat content [33]. Although liver biopsy is the gold standard for NAFLD diagnosis, it has drawbacks such as invasive nature, sampling inconsistency, and low acceptance by patients and is unsuitable for longitudinal monitoring. Moreover, it may not be appropriate to perform liver biopsies in low-risk subjects; biopsy-proven NAFLD patients may not be representative of the general population with NAFLD. Non-invasive biomarkers have been suggested for the diagnosis of NASH and fibrosis [33], and biopsies are reserved for individuals with NASH diagnosis based on imaging and non-invasive assessments [34]. The use of various modalities and cut-offs to establish the severity of steatosis or fibrosis can affect the overall assessment; thus, a common method should be standardized.

Few meta-analyses were conducted to evaluate the relationship between coffee and NAFLD. In a meta-analysis of four cross-sectional studies and two case-control studies, no association was found between total caffeine consumption ( $\mathrm{mg}$ /day) with either the prevalence or the risk of liver fibrosis related to NAFLD. However, when stratified by the type of caffeine intake, regular coffee caffeine intake (mg/day) significantly decreased the risk of liver fibrosis or development of NAFLD [35]. Lower risk and prevalence of NAFLD in coffee drinkers and the protective effect of coffee on liver fibrosis have also been reported in other studies [36-38]. In comparison to these meta-analyses, we included a larger number and more recent studies in the analysis. In contrast to prior studies, we specifically investigated the association between coffee consumption and NAFLD incidence. We also conducted a subgroup analysis by study design, questionnaire administration, study quality, energy intake adjustment, and population of interest which has not been previously performed. A systematic review by Sewter et al. included studies assessing the progression of NAFLD to hepatic fibrosis and/or cirrhosis in relation to coffee consumption [38]; however, our study aimed to conduct a meta-analysis to determine the impact of coffee consumption on NAFLD incidence, prevalence, and fibrosis risk in the general population or patients with NAFLD. Contrary to the systematic review by Sewter et al., studies without detailed information for risk estimates were excluded from our meta-analysis.

Some limitations should be noted in interpreting the results of the current metaanalysis. First, this analysis was limited to the English language and exclusion of manuscripts in other languages or unpublished studies might contribute to publication bias. The majority of the studies in this area were cross-sectional studies showing an association but not able to determine causality and limiting the capability to evaluate chronological associations. Reverse causality is a common concern in cross-sectional studies as the predictor and outcome are assessed at the same time. The risk of bias and the quality of the studies were assessed using the NOS, a commonly used tool for observational studies. Although a conventional sampling method was applied in most of the studies, comparison between responders and non-responders was not reported, contributing to self-selection bias.

Observational studies are limited by the inability to account for confounding factors, including nutritional and lifestyle behaviors (diet, physical activity, smoking, obesity, insulin resistance, and body fat distribution). Several comorbidities affecting the severity of NAFLD such as diabetes and obesity differ between coffee drinkers and non-drinkers, and a lack of adjustment can lead to bias in the assessment of the effect of the exposure. Daily energy intake may be higher in subjects who regularly drink coffee with additives such as sugar and cream [39], and without adjusting for daily calorie intake, it is impossible to properly establish the association between coffee intake and NAFLD. Prospective studies should evaluate dietary habits and medications that might impact liver fibrosis such as thiazolidinediones, statins, vitamin E, and omega 3 as potential confounders [40]. In this meta-analysis, the association between coffee consumption and liver fibrosis remained 
significant in studies adjusting for calorie intake and other confounders such as age, sex, BMI, HOMA-IR, and physical activity.

One of the main challenges in these studies is to properly assess an individual patient's coffee consumption. Coffee intake was evaluated using a questionnaire at a single timepoint and therefore the impact of changes in habitual coffee intake was not determined. The validity of the questionnaire and blinded interview are also important considerations. Retrospective self-reported questionnaires infer the risk of recall or reporting bias [41]. Illustrative exemplification of standard drinks/coffee cups is another approach to mitigate error in estimating drink size [42] which needs to be considered in future studies. Classification of regular coffee intake differed between studies, and the unit was not standardized across included studies. Therefore, the optimal dose that correlates with the greatest reduced risk would need to be established in future clinical trials.

Potential beneficial components of regular coffee need to be determined in prospective studies as coffee contains several compounds including caffeine, polyphenol (chlorogenic acid), and diterpenes (cafestol and kahweol) [43]. Experimental studies show coffee mitigates hepatic fibrosis by down-regulating profibrogenic genes [44,45], inhibiting adhesion and activation of hepatic stellate cells [12], and activating nuclear factor erythroid 2-related factor 2, inducing the antioxidant enzymes system [45]. It was also suggested that coffee attenuates hepatic steatosis through upregulation of $\beta$-oxidation, downregulation of de novo lipogenesis, suppression of oxidative stress [46], and prevention of adipogenic hepatic steatosis [47]. The antioxidant properties of caffeine [48] and the inhibitory effect of kahweol on hepatic inflammation [49] may help to attenuate the development of NASH (Supplementary Figure S1). A recent meta-analysis of randomized clinical trials suggests the anti-inflammatory impact of coffee might be related to its ability to lower tumor necrosis factor alfa levels. There was no significant association with other markers including interleukin -6 and C-reactive protein in this meta-analysis [50].

\section{Conclusions}

This meta-analysis confirms the protective role of regular coffee consumption on the risk of significant liver fibrosis. No association was observed between coffee consumption and NAFLD incidence or prevalence. In the absence of approved drug therapy, coffee should be recommended in conjunction with lifestyle changes. The threshold of coffee consumption to achieve its protective effect needs to be established in prospective trials. It is still unclear which element of coffee confers benefit. Prospective studies with a comprehensive evaluation of coffee intake over time will be important to clarify the amount, type, preparation method, and pattern of coffee consumption that has the most benefit in liver disease. The impact of possible confounding factors such as enhanced nutritional status or physical activity cannot be excluded.

Supplementary Materials: The following are available online at https:/ /www.mdpi.com/article/10 .3390/nu13093042/s1, Figure S1: Mechanisms by which coffee impacts hepatic steatosis and fibrosis; Table S1: PRISMA (Preferred Reporting Items for Systematic reviews and Meta-Analysis).

Author Contributions: Research and selection of abstracts, review of the full-length articles, data extraction and analysis, and writing the final version of the manuscript were performed by M.E. and A.J.M.-L.; R.A.B. and S.I. assisted with writing and reviewing the manuscript. All authors have commented on the manuscript and approved the final version. All authors have read and agreed to the published version of the manuscript.

Funding: This research received no external funding.

Data Availability Statement: The data presented in this study are available in Tables 1 and 2.

Acknowledgments: Maryam Ebadi has been awarded the Canadian Institutes of Health Research (CIHR) - Institute of Nutrition, Metabolism, and Diabetes (INMD) Fellowship-Hepatology, in partnership with the Canadian Association for the Study of the Liver (CASL) and the Canadian Liver Foundation (CLF) (HGY-164788). Maryam Ebadi and Aldo J. Montano-Loza have been awarded 
the Canadian National Transplant Research Program (CNTRP), the Alberta Transplant Institute Foundation (ATIF) Innovation Grant Award 2018, the University of Alberta Hospital Foundation (UHF) Grant 2018, and the Canadian Liver Foundation (CLF) Grant 2019.

Conflicts of Interest: The authors declare no conflict of interest.

\section{References}

1. $\quad$ Eslam, M.; Newsome, P.N.; Sarin, S.K.; Anstee, Q.M.; Targher, G.; Romero-Gomez, M.; Zelber-Sagi, S.; Wong, V.W.-S.; Dufour, J.-F.; Schattenberg, J.M.; et al. A new definition for metabolic dysfunction-associated fatty liver disease: An international expert consensus statement. J. Hepatol. 2020, 73, 202-209.

2. Eslam, M.; Sanyal, A.J.; George, J. International Consensus Panel. MAFLD: A Consensus-Driven Proposed Nomenclature for Metabolic Associated Fatty Liver Disease. Gastroenterology 2020, 158, 1999-2014.e1.

3. Vernon, G.; Baranova, A.; Younossi, Z.M. Systematic review: The epidemiology and natural history of non-alcoholic fatty liver disease and non-alcoholic steatohepatitis in adults. Aliment. Pharmacol. Ther. 2011, 34, 274-285.

4. Wong, R.J.; Aguilar, M.; Cheung, R.; Perumpail, R.B.; Harrison, S.A.; Younossi, Z.M.; Ahmed, A. Nonalcoholic steatohepatitis is the second leading etiology of liver disease among adults awaiting liver transplantation in the United States. Gastroenterology 2015, 148, 547-555.

5. Tesfay, M.; Goldkamp, W.J.; Neuschwander-Tetri, B.A. NASH: The Emerging Most Common Form of Chronic Liver Disease. Mol. Med. 2018, 115, 225-229.

6. Taylor, R.S.; Taylor, R.J.; Bayliss, S.; Hagström, H.; Nasr, P.; Schattenberg, J.M.; Ishigami, M.; Toyoda, H.; Wong, V.W.-S.; Peleg, N.; et al. Association Between Fibrosis Stage and Outcomes of Patients With Nonalcoholic Fatty Liver Disease: A Systematic Review and Meta-Analysis. Gastroenterology 2020, 158, 1611-1625.e12.

7. Armstrong, M.; Houlihan, D.D.; Bentham, L.; Shaw, J.C.; Cramb, R.; Olliff, S.; Gill, P.S.; Neuberger, J.M.; Lilford, R.J.; Newsome, P. Presence and severity of non-alcoholic fatty liver disease in a large prospective primary care cohort. J. Hepatol. 2012, 56, 234-240.

8. Chalasani, N.; Younossi, Z.; LaVine, J.E.; Charlton, M.; Cusi, K.; Rinella, M.; Harrison, S.A.; Brunt, E.M.; Sanyal, A.J. The diagnosis and management of nonalcoholic fatty liver disease: Practice guidance from the American Association for the Study of Liver Diseases. Hepatology 2017, 67, 328-357.

9. Molloy, J.W.; Calcagno, C.J.; Williams, C.D.; Jones, F.J; Torres, D.M.; Harrison, S.A. Association of coffee and caffeine consumption with fatty liver disease, nonalcoholic steatohepatitis, and degree of hepatic fibrosis. Hepatology 2011, 55, $429-436$.

10. Liu, R.; Guo, X.; Park, Y.; Huang, X.; Sinha, R.; Freedman, N.D.; Hollenbeck, A.R.; Blair, A.; Chen, H. Caffeine Intake, Smoking, and Risk of Parkinson Disease in Men and Women. Am. J. Epidemiol. 2012, 175, 1200-1207.

11. Bambha, K.; Wilson, L.A.; Unalp, A.; Loomba, R.; Neuschwander-Tetri, B.A.; Brunt, E.M.; Bass, N.M.; The Nonalcoholic Steatohepatitis Clinical Research Network (NASH CRN). Coffee consumption in NAFLD patients with lower insulin resistance is associated with lower risk of severe fibrosis. Liver Int. 2014, 34, 1250-1258.

12. Shim, S.G.; Jun, D.W.; Kim, E.K.; Saeed, W.K.; Lee, K.N.; Lee, H.L.; Lee, O.Y.; Choi, H.S.; Yoon, B.C. Caffeine attenuates liver fibrosis via defective adhesion of hepatic stellate cells in cirrhotic model. J. Gastroenterol. Hepatol. 2013, 28, $1877-1884$.

13. Arauz, J.; Zarco, N.; Segovia, J.; Shibayama, M.; Tsutsumi, V.; Muriel, P. Caffeine prevents experimental liver fibrosis by blocking the expression of TGF-beta. Eur. J. Gastroenterol. Hepatol. 2014, 26, 164-173.

14. Li, S.; Tan, H.Y.; Wang, N.; Cheung, F.; Hong, M.; Feng, Y. The Potential and Action Mechanism of Polyphenols in the Treatment of Liver Diseases. Oxidative Med. Cell. Longev. 2018, 2018, 8394818.

15. Vitaglione, P.; Morisco, F.; Mazzone, G.; Amoruso, D.C.; Ribecco, M.T.S.; Romano, A.; Fogliano, V.; Caporaso, N.; D’Argenio, G. Coffee reduces liver damage in a rat model of steatohepatitis: The underlying mechanisms and the role of polyphenols and melanoidins. Hepatology 2010, 52, 1652-1661.

16. Imatoh, T.; Kamimura, S.; Miyazaki, M. Coffee but not green tea consumption is associated with prevalence and severity of hepatic steatosis: The impact on leptin level. Eur. J. Clin. Nutr. 2015, 69, 1023-1027.

17. Stang, A. Critical evaluation of the Newcastle-Ottawa scale for the assessment of the quality of nonrandomized studies in meta-analyses. Eur. J. Epidemiol. 2010, 25, 603-605.

18. Herzog, R.; Álvarez-Pasquin, M.J.; Díaz, C.; Del Barrio, J.L.; Estrada, J.M.; Gil, Á. Are healthcare workers' intentions to vaccinate related to their knowledge, beliefs and attitudes? a systematic review. BMC Public Health 2013, 13, 154.

19. Higgins, J.P.T.; Thompson, S.G.; Deeks, J.J.; Altman, D.G. Measuring inconsistency in meta-analyses. BMJ 2003, 327, 557-560.

20. Egger, M.; Smith, G.D.; Schneider, M.; Minder, C. Bias in meta-analysis detected by a simple, graphical test. BMJ 1997, 315, 629-634.

21. Chung, H.-K.; Nam, J.S.; Lee, M.-Y.; Kim, Y.-B.; Won, Y.-S.; Song, W.-J.; Kim, Y.-H.; Ahn, C.W.; Sung, K.-C. The increased amount of coffee consumption lowers the incidence of fatty liver disease in Korean men. Nutr. Metab. Cardiovasc. Dis. 2020, 30, $1653-1661$.

22. Funatsu, K.; Yamashita, T.; Nakamura, H. Coffee Consumption is Associated with a Lower Incidence of Fatty Liver in Middle-aged Men. J. Health Sci. 2011, 57, 406-413.

23. Zelber-Sagi, S.; Salomone, F.; Webb, M.; Lotan, R.; Yeshua, H.; Halpern, Z.; Santo, E.; Oren, R.; Shibolet, O. Coffee consumption and nonalcoholic fatty liver onset: A prospective study in the general population. Transl. Res. 2015, 165, 428-436.

24. Veronese, N.; Notarnicola, M.; Cisternino, A.M.; Reddavide, R.; Inguaggiato, R.; Guerra, V.; Rotolo, O.; Zinzi, I.; Leandro, G.; Correale, M.; et al. Coffee Intake and Liver Steatosis: A Population Study in a Mediterranean Area. Nutrients 2018, 10, 89. 
25. Alferink, L.J.; Fittipaldi, J.; Jong, J.C.K.-D.; Taimr, P.; Hansen, B.E.; Metselaar, H.J.; Schoufour, J.D.; Ikram, M.A.; Janssen, H.L.; Franco, O.; et al. Coffee and herbal tea consumption is associated with lower liver stiffness in the general population: The Rotterdam study. J. Hepatol. 2017, 67, 339-348.

26. Graeter, T.; Niedermayer, P.C.; Mason, R.A.; Oeztuerk, S.; Haenle, M.M.; Koenig, W.; Boehm, B.O.; Kratzer, W.; EMIL-Study group. Coffee consumption and NAFLD: A community based study on 1223 subjects. BMC Res. Notes 2015, 8, 640.

27. Katsagoni, C.N.; Georgoulis, M.; Papatheodoridis, G.; Fragopoulou, E.; Ioannidou, P.; Papageorgiou, M.; Alexopoulou, A.; Papadopoulos, N.; Deutsch, M.; Kontogianni, M. Associations Between Lifestyle Characteristics and the Presence of Nonalcoholic Fatty Liver Disease: A Case-Control Study. Metab. Syndr. Relat. Disord. 2017, 15, 72-79.

28. Zhang, X.; Goh, G.B.; Chan, W.; Wong, G.L.; Fan, J.; Seto, W.; Huang, Y.; Lin, H.; Lee, I.; Lee, H.W.; et al. Unhealthy lifestyle habits and physical inactivity among Asian patients with non-alcoholic fatty liver disease. Liver Int. 2020, 40, $2719-2731$.

29. Anty, R.; Marjoux, S.; Iannelli, A.; Patouraux, S.; Schneck, A.-S.; Bonnafous, S.; Gire, C.; Amzolini, A.; Ben-Amor, I.; Saint-Paul, M.-C.; et al. Regular coffee but not espresso drinking is protective against fibrosis in a cohort mainly composed of morbidly obese European women with NAFLD undergoing bariatric surgery. J. Hepatol. 2012, 57, 1090-1096.

30. Gutiérrez-Grobe, Y.; Chavez-Tapia, N.; Sánchez-Valle, V.; Gavilanes-Espinar, J.G.; Ponciano-Rodríguez, G.; Uribe, M.; MéndezSánchez, N. High coffee intake is associated with lower grade nonalcoholic fatty liver disease: The role of peripheral antioxidant activity. Ann. Hepatol. 2012, 11, 350-355.

31. Chen, Y.-P.; Lu, F.-B.; Hu, Y.-B.; Xu, L.-M.; Zheng, M.-H.; Hu, E.-D. A systematic review and a dose-response meta-analysis of coffee dose and nonalcoholic fatty liver disease. Clin. Nutr. 2019, 38, 2552-2557.

32. Mishra, P.; Younossi, Z.M. Abdominal ultrasound for diagnosis of nonalcoholic fatty liver disease (NAFLD). Am. J. Gastroenterol. 2007, 102, 2716-2717.

33. Castera, L.; Friedrich-Rust, M.; Loomba, R. Noninvasive Assessment of Liver Disease in Patients with Nonalcoholic Fatty Liver Disease. Gastroenterology 2019, 156, 1264-1281.e4.

34. Chartampilas, E. Imaging of nonalcoholic fatty liver disease and its clinical utility. Hormones 2018, 17, 69-81.

35. Shen, H.; Rodriguez, A.C.; Shiani, A.; Lipka, S.; Shahzad, G.; Kumar, A.; Mustacchia, P. Association between caffeine consumption and nonalcoholic fatty liver disease: A systemic review and meta-analysis. Ther. Adv. Gastroenterol. 2016, 9, 113-120.

36. Marventano, S.; Salomone, F.; Godos, J.; Pluchinotta, F.R.; Del Rio, D.; Mistretta, A.; Grosso, G. Coffee and tea consumption in relation with non-alcoholic fatty liver and metabolic syndrome: A systematic review and meta-analysis of observational studies. Clin. Nutr. 2016, 35, 1269-1281.

37. Wijarnpreecha, K.; Thongprayoon, C.; Ungprasert, P. Coffee consumption and risk of nonalcoholic fatty liver disease: A systematic review and meta-analysis. Eur. J. Gastroenterol. Hepatol. 2017, 29, e8-e12.

38. Sewter, R.; Heaney, S.; Patterson, A. Coffee Consumption and the Progression of NAFLD: A Systematic Review. Nutrients 2021, $13,2381$.

39. Kim, H.-J.; Cho, S.; Jacobs, D.R., Jr.; Park, K. Instant coffee consumption may be associated with higher risk of metabolic syndrome in Korean adults. Diabetes Res. Clin. Pract. 2014, 106, 145-153.

40. Caldwell, S. NASH Therapy: Omega 3 supplementation, vitamin E, insulin sensitizers and statin drugs. Clin. Mol. Hepatol. 2017, 23, 103-108.

41. Althubaiti, A. Information bias in health research: Definition, pitfalls, and adjustment methods. J. Multidiscip. Healthc. 2016, 9 , 211-217.

42. Stockwell, T.; Donath, S.; Cooper-Stanbury, M.; Chikritzhs, T.; Catalano, P.; Mateo, C. Under-reporting of alcohol consumption in household surveys: A comparison of quantity-frequency, graduated-frequency and recent recall. Addiction 2004, 99, $1024-1033$.

43. Alferink, L.J.M.; Jong, J.C.K.-D.; Murad, S.D. Potential Mechanisms Underlying the Role of Coffee in Liver Health. Semin. Liver Dis. 2018, 38, 193-214.

44. Arauz, J.; Moreno, M.G.; Cortés-Reynosa, P.; Salazar, E.P.; Muriel, P. Coffee attenuates fibrosis by decreasing the expression of TGF-beta and CTGF in a murine model of liver damage. J. Appl. Toxicol. 2013, 33, 970-979.

45. Gordillo-Bastidas, D.; Oceguera-Contreras, E.; Salazar-Montes, A.; González-Cuevas, J.; Hernández-Ortega, L.D.; ArmendárizBorunda, J. Nrf2 and Snail-1 in the prevention of experimental liver fibrosis by caffeine. World J. Gastroenterol. 2013, 19, 9020-9033.

46. Helal, M.G.; Ayoub, S.E.; Elkashefand, W.F.; Ibrahim, T.M. Caffeine affects HFD-induced hepatic steatosis by multifactorial intervention. Hum. Exp. Toxicol. 2018, 37, 983-990.

47. Takahashi, S.; Egashira, K.; Saito, K.; Jia, H.; Abe, K.; Kato, H. Coffee intake down-regulates the hepatic gene expression of peroxisome proliferator-activated receptor gamma in C57BL/6J mice fed a high-fat diet. J. Funct. Foods 2014, 6, $157-167$.

48. Metro, D.; Cernaro, V.; Santoro, D.; Papa, M.; Buemi, M.; Benvenga, S.; Manasseri, L. Beneficial effects of oral pure caffeine on oxidative stress. J. Clin. Transl. Endocrinol. 2017, 10, 22-27.

49. Seo, H.Y.; Kim, M.K.; Lee, S.H.; Hwang, J.S.; Park, K.G.; Jang, B.K. Kahweol Ameliorates the Liver Inflammation through the Inhibition of NF-kappaB and STAT3 Activation in Primary Kupffer Cells and Primary Hepatocytes. Nutrients 2018, $10,863$.

50. Asbaghi, O.; Kashkooli, S.; Mardani, M.; Kelishadi, M.R.; Fry, H.; Kazemi, M.; Kaviani, M. Effect of green coffee bean extract supplementation on liver function and inflammatory biomarkers: A meta-analysis of randomized clinical trials. Complementary Ther. Clin. Pract. 2021, 43, 101349. 